Superconducting Super Collider Laboratory

草

\title{
Luminosity Dilution Due to Random Offset Beam-Beam Interaction
}

\section{G. Stupakov}

November 1991 


\title{
Luminosity Dilution Due to Random Offset Beam-Beam Interaction
}

\author{
G. Stupakov* \\ Accelerator Design and Operations Division \\ Superconducting Super Collider Laboratory ${ }^{\dagger}$ \\ 2550 Beckleymeade Avenue \\ Dallas, TX 75237
}

November 1991

DISCLAIMER

This report was prepared as an account of work sponsored by an agency of the Uniled States Government. Neither the United States Government nor any agency thereof, nor any of their employees. makes any warranty, express or implied, or as sumes any legal liability or responsibility for the accuracy. completeness. or usefulness of any info mation, apparatus, product, or process disclosed. or represents that its use would not infringe privately owned rights Reference herein to any specific commercial product. process, or service by trade name, trademark. manufacturer. or otherwise does not necessarily constitute or imply its endorsement, recommendation. or favoring by the United States Government or any agency thereof. The views and opinions of authors expressed herein do not necessarily state or reflect those of the United States Government or any agency thereof.

\footnotetext{
* Guest scientist visiting from the Institute of Nuclear Physics, Novosibirsk, 630090, USSR.

' Operated by the Universities Research Association, Inc., for the U.S. Department of Energy' under Contract No. DE-AC35-89ER40486.

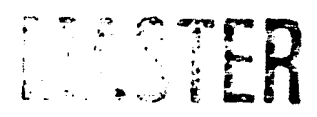




\title{
Luminosity Dilution Due to Random Offset Beam-Beam Interaction
}

\author{
G. Stupakov
}

\begin{abstract}
We consider beam-beam interaction in a collider in the case when the beams randomly displace around the equilibrium orbit at the interaction point. Due to the random part of the interaction, particles diffuse over the betatron amplitude causing an emittance growth of the beam. A Fokker-Planck equation is derived in which a diffusion coefficient is related with the spectral density of the noise. Estimations for the Superconducting Super Collider parameters give a tolerable level of the high-frequency beam offset at the interaction point.
\end{abstract}




\section{CONTENTS}

$1.0 \quad$ INTRODUCTION

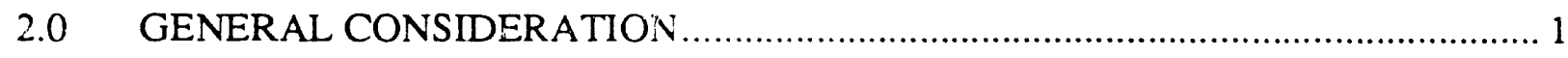

3.0 AMPLITUDE AND PHASE CHANGE DUE TO MULTIPLE INTERACTIONS OF THE BUNCHES ……………….................................... 2

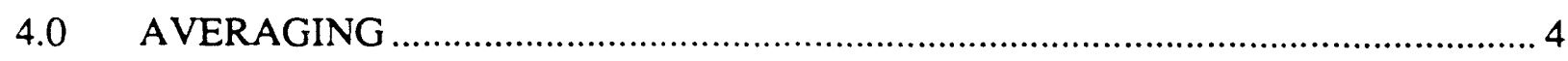

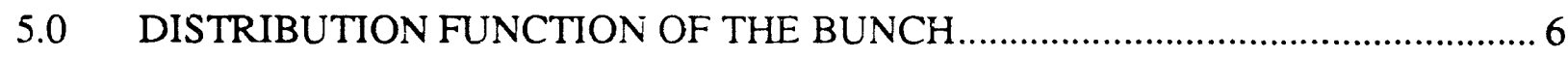

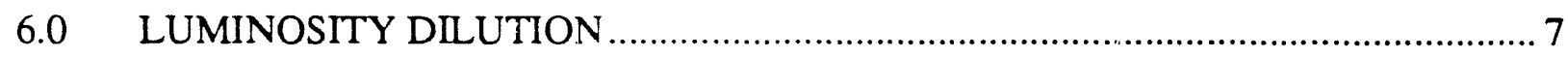

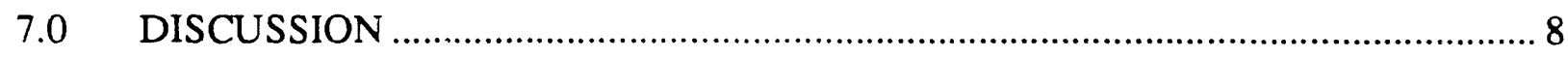

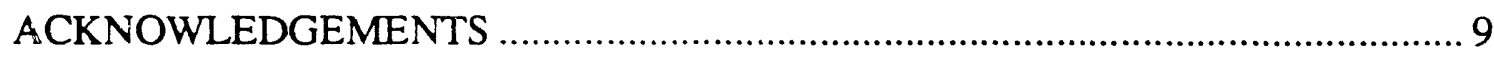

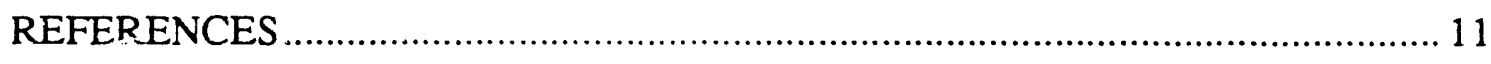

APPENDIX A ………

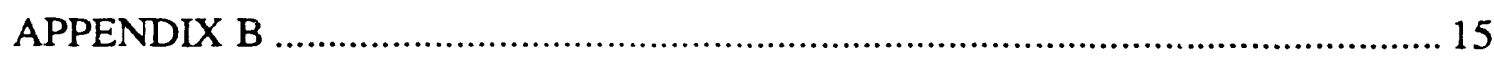




\subsection{INTRODUCTION}

The required confinement time of protons in the Superconducting Super Collider (SSC) (10-20 hours) ${ }^{1}$ makes it necessary to carefully study even small effects that can gradually dilute the luminosity of the collider over a long time. A possible physical mechanism which can influence the beam performance is related to different types of noise in the machine. These include ground motion causing random displacements of magnets, sound vibrations, and if noise. These noises could excite betatron and synchrotron oscillations in the beams over an allowable level and result in the growth of the emittance of the beams.

A particular mechanism of beam degradation that we study in this paper is related to random beam displacements at the interaction points caused by noisy betatron oscillations of the bunches around the equilibrium orbits. We do not specify here the physical mechanism which drives these oscillations, but we do make some natural assumptions about statistical properties of the amplitudes of the oscillations. Due to nonlinearity of the beam-beam interaction, the random setoffs of the interacting beams result in a diffusion process that increases the radial extension of the beams and, hence, decreases the luminosity. The main purpose of this paper is to find constraints on the noise level (in terms of the beam offset at the interaction point) at which the effect of the luminosity degradation is not imponant for the SSC.

\subsection{GENERAL CONSIDERATION}

We consider a simple model in which two bunches are collided periodically at one interaction point. For the sake of simplicity we choose a one-dimensional model, assuming that the width of the bunch in the $z$-direction is much larger than its dimension in the $x$-direction, that is $\sigma_{x}$ « $\sigma_{z}$. We will also neglect the influence of one beam upon the other (the weak-strong beam model), assuming that one of the colliding beams (a strong one) has a fixed distribution of particles over the betatron amplitudes and phases, and that this distribution function is not changing in time. However, the beam-beam interactions evolve the distribution function of the other beam, and the problem consists in describing its evolution.

Throughout this paper we will use dimensionless units normalizing the length on $\sigma_{x}$, momentum on $P \sigma_{x^{\prime}} \beta^{*}\left(P\right.$ is the particles' momentum, $\beta^{*}$ is the value of the $\beta$ function at the interaction point), and action on $P \sigma_{x}^{2} / \beta^{*}$.

The initial density profile $n_{0}(x)$ of the bunch in the $x$-direction is assumed to be a Gaussian:

$$
n_{0}(x)=\frac{N_{\mathrm{B}}}{\sqrt{2 \pi} l} \mathrm{e}^{-x^{2} / 2},
$$

where $l$ is the bunch length and $N_{B}$ is the number of particles in the bunch per unit length in the z-direction. In Eq. (1) we assume a step-like density distribution in the bunch along its orbit.

To describe betatron oscillations, we use canonically conjugate action $J$ and phase $\phi$ variables. The phase $\phi$ is chosen so that it is constant for the unperturbed (that is, neglecting the beam-beam interaction) betatron motion, $\phi=$ const, so that in our dimensionless units,

$$
x=\sqrt{2 J \beta / \beta^{*}} \sin (v \theta+\varphi(\theta)+\phi),
$$

where $v$ is the betatron tune, and $\varphi(\theta)$ is the periodic part of the betatron phase, $\varphi(\theta)=\varphi(\theta+2 \pi)$. For what follows, we will need to evaluate $x$ at the interaction point only where $\beta=\beta^{*}$ and $\varphi$ can be chosen to be zero. At this point, Eq. (2) takes the form

$$
x=\sqrt{2} \sin (v \theta+\phi) .
$$


The initial distribution of particles of the bunch in the action variable is given by a function $F_{0}(J)$. One can easily check that the distribution function which corresponds to the given density profile, Eq. (1), has the following form:

$$
F_{0}(J)=\frac{1}{2 \pi} \mathrm{e}^{-J}
$$

It is normalized so that $\int_{0}^{\infty} d J \int_{0}^{2 \pi} d \phi F_{0}=1$.

In the next sections we will see that due to the beam-beam interaction of the beams with a random setoff, the distribution function $F$ gets a perurbation around its equilibrium value, $F=F_{0}(J)+\Delta F(J)$. (We will show later that an average $\Delta F$ does not depend on $\phi$.) The density perturbation $\Delta n(x)$ associated with $\Delta F$ is given by

$$
\begin{aligned}
\Delta \mathrm{n}(\mathrm{x}) & \left.=\frac{N_{\mathrm{B}}}{l} \int_{0}^{\infty} d J j d \phi \Delta F(J) \delta x-\sqrt{2 J} \sin (v \theta+\phi)\right]= \\
& =\frac{2 N_{\mathrm{B}}}{l} \underset{x^{2} / 2}{\infty} \int d J \frac{\Delta F(J)}{\sqrt{2 J-x^{2}}} .
\end{aligned}
$$

Because the density of the bunch differs from the initial one, the luminosity of the machine also changes. Its relative change, $\Delta L L_{0}$, is

$$
\frac{\Delta L}{L_{0}}=\frac{\int n_{0} \Delta n(x) d x}{\int n_{0}^{2} d x}=\frac{1}{\sqrt{\pi}} \int_{-\infty}^{\infty} x^{2} \frac{\Delta n}{n_{0}} d x .
$$

Putting Eqs. (1) and (5) into Eq. (6) and performing the integration over $x$, one finds

$$
\frac{\Delta L}{L_{0}}=2^{32} \pi \int_{0}^{\infty} d J \Delta F(J) \mathrm{e}^{-J / 2} \mathrm{I}_{0}(J / 2) d J
$$

where $\mathrm{I}_{0}$ is the modified Bessel function of the zeroth order.

Now, the problem is to find the perurbation of the distribution function $\Delta F$ and to evaluate the integral (7). In the next three sections we compute $\Delta F$, assuming that it is small compared with $F_{0}$, $\Delta F_{\kappa} F_{0}$. With this assumption, we will be able to consider an initial stage of the degradation of the luminosity when $\Delta L$ (being negative) linearly grows in time. Though not valid on a long time scale, this approach allows us to predict the strength of the effect and to qualitatively estimate its danger for a real machine.

\subsection{AMPLITUDE AND PHASE CHANGE DUE TO MULTIPLE INTERACTIONS OF THE BUNCHES}

A dimensir iess potential energy $V_{\mathrm{m}}$ governing the interaction of the two colliding bunches with their centers displ; ed by a distance $\zeta_{\mathrm{m}}$ in the $x$-direction is given by (see, for example, Reference 2 ):

$$
V_{\mathrm{m}}=4 \pi \xi h\left(x-\zeta_{\mathrm{m}}\right) \delta(\theta-2 \pi(m-1)) \text {. }
$$


where $\xi$ is the conventional interaction parameter:

$$
\xi=\frac{N_{\mathrm{B}} r_{\mathrm{p}} \beta^{*}}{\sqrt{2 \pi \sigma \gamma}}
$$

( $r_{\mathrm{p}}$ is the classical proton radius) and the function $h(x)$ is

$$
h(x)=\int_{0}^{x} d y \int_{0}^{y} d z e^{-z^{2}}
$$

The subscript $m$ denotes the collision number, $m=1,2 \ldots$, and we assume that the first interaction occurs at $\theta=0$.

The effects under consideration are related to small, $\zeta_{\mathrm{m}} * 1$, random setoffs of the beams with respect to each other. For small $\zeta_{m}$, the function $h\left(x-\zeta_{m}\right)$ can be expanded in a power series. Keeping only the first two terms in this series, we have

$$
h\left(x-\zeta_{\mathrm{m}}\right)=h(x)-\zeta_{\mathrm{m}} h^{\prime}(x)
$$

where the prime denotes derivative with respect to $x$. The first term on the right side of Eq. (11) refers to the head-on interaction of the beams. For small $\xi$, the main effect of this interaction is a non-linear tune shift, $\Delta v$, that can be included in $v$. For our purposes, the crucial one is the second term in Eq. (11), with the corresponding contribution $\widetilde{V}_{\mathrm{m}}$ to the potential eriergy given by

$$
\widetilde{V}_{\mathrm{m}}=\widetilde{v}_{\mathrm{m}} \delta[\theta-2 \pi(m-1)] \text {. }
$$

where

$$
\tilde{v}_{\mathrm{m}}=4 \pi \xi \zeta_{\mathrm{m}} h^{\prime}(x)
$$

The term $\widetilde{V}_{\mathrm{m}}$ in the Hamiltonian produces perturbations $\delta J_{\mathrm{m}}$ and $\delta \phi_{\mathrm{m}}$ of action and phase in the $m$ th interaction of the bunches. These can be found from the Hamiltonian equations of motion. A simple integration yields

$$
\begin{aligned}
\delta J_{\mathrm{m}}=-\frac{\partial \tilde{v}_{\mathrm{m}}}{\partial \phi}= & -4 \pi \xi \zeta_{\mathrm{m}} h^{\prime \prime}\left\{\sqrt{2 J_{\mathrm{m}-1}} \sin \left[2 \pi v(m-1)+\phi_{\mathrm{m}-1}\right]\right\} \times \\
& \times \sqrt{2 J_{\mathrm{m}-1}} \cos \left[2 \pi v(m-1)+\phi_{\mathrm{m}-1}\right], \\
\delta \phi_{\mathrm{m}}=\frac{\partial \tilde{v}_{\mathrm{m}}}{\bar{\partial} J}= & 4 \pi \xi \zeta_{\mathrm{m}} h^{\prime \prime}\left\{\left(\sqrt{2 J_{\mathrm{m}-1}} \sin [2 \pi \mathrm{vm}-1)+\phi_{\mathrm{m}-1}\right]\right\} \times \\
& \times \frac{1}{\sqrt{2 J_{\mathrm{m}-1}}} \sin \left[2 \pi v(\mathrm{~m}-1)+\phi_{\mathrm{m}-1}\right],
\end{aligned}
$$

where $J_{\mathrm{m}-1}$ and $\phi_{\mathrm{m}-1}$ denote the value of $J$ and $\phi$ before the $m$ th interaction. Total change of $J$ and $\phi$ resulting from $M$ interactions, $\Delta J_{M}$ and $\Delta \phi_{M}$. can be found by summing Eqs. (14) and (15) for $m=1,2, \ldots M$ : 


$$
\begin{aligned}
& \Delta J_{\mathrm{M}}=-4 \pi \xi \sum_{m=0}^{m=M-1} \zeta_{\mathrm{m}} \sqrt{2 J_{\mathrm{m}}} h^{\prime \prime}\left[\sqrt{2 \mathrm{~J}_{\mathrm{m}}} \sin \left(2 \pi v m+\phi_{\mathrm{m}}\right)\right] \cos \left(2 \pi v m+\phi_{\mathrm{m}}\right) \\
& \Delta \phi_{\mathrm{M}}=-4 \pi \xi \sum_{m=0}^{m=M-1} \zeta_{\mathrm{m}} \frac{1}{\sqrt{2 J_{\mathrm{m}}}} h^{\prime \prime}\left[\sqrt{2 J_{\mathrm{m}}} \sin \left(2 \pi v m+\phi_{\mathrm{m}}\right)\right] \cos \left(2 \pi v m+\phi_{\mathrm{m}}\right) .
\end{aligned}
$$

Using the explicit expression $h^{\prime \prime}(x)=\exp \left(-x^{2} / 2\right)$ (see Eq. (10)), one can expand the exponential into a Fourier series:

$$
\mathrm{e}^{-x^{2}}=\mathrm{e}^{-J \sin ^{2}(2 \pi v m+\phi)}=\mathrm{e}^{-J / 2}\left[\mathrm{I}_{0}\left(\frac{J}{2}\right)+2 \sum_{k=1}^{\infty} \mathrm{I}_{k}\left(\frac{J}{2}\right) \cos 2 k(2 \pi v m+\phi)\right]
$$

where $\mathrm{I}_{\mathrm{k}}$ denotes the modified Bessel function of $k$ th order. Putting Eq. (18) into Eqs. (16) and (17), we find

$$
\begin{aligned}
& \Delta J_{\mathrm{M}}=-4 \pi \xi \sum_{m=0}^{m=M-1} \zeta_{\mathrm{m}} \sqrt{2 J_{\mathrm{m}}} \mathrm{e}^{-J_{\mathrm{m}}} \sum_{k=0}^{\infty}\left[\mathrm{I}_{\mathrm{k}}\left(\frac{J_{\mathrm{m}}}{2}\right)+\mathrm{I}_{\mathrm{k}+1}\left(\frac{J_{\mathrm{m}}}{2}\right)\right] \cos \left[(2 k+1)\left(2 \pi v m+\phi_{\mathrm{m}}\right)\right], \\
& \Delta \phi_{\mathrm{M}}=4 \pi \xi \sum_{m=0}^{m=M-1} \zeta_{\mathrm{m}} \mathrm{e}^{-J_{\mathrm{m}} / 2} \frac{1}{\sqrt{2 J_{\mathrm{m}}}} \sum_{k=0}^{\infty}\left[\mathrm{I}_{\mathrm{k}}\left(\frac{J_{\mathrm{m}}}{2}\right)+\mathrm{I}_{\mathrm{k}+1}\left(\frac{J_{\mathrm{m}}}{2}\right)\right] \sin \left[(2 k+1)\left(2 \pi v m+\phi_{\mathrm{m}}\right)\right] .
\end{aligned}
$$

Now let us take into account the smallness of the parameter $\xi \zeta$ in Eqs. (19) and (20) and consider a case when $M » 1$ but $\Delta U_{\mathrm{m}}, \Delta \phi_{\mathrm{m}}$ \&l. In other words, the changes of the action and phase (and hence the distribution function and the luminosity) are assumed to be relatively small on the time interval under consideration. In linear approximation in the parameter $\xi \zeta$ one can substitute $f_{n}$ and $\phi_{\mathrm{m}}$ by $J_{0}$ and $\phi_{0}$ on the right side of Eqs. (19) and (20):

$$
\Delta J_{M}=-4 \pi \xi \sum_{m=0}^{m=M-1} \zeta_{m} \sqrt{2 J_{0}} \mathrm{e}^{-J_{0} / 2} \sum_{k=0}^{\infty}\left[\mathrm{I}_{\mathrm{k}}\left(\frac{J_{0}}{2}\right)+\mathrm{I}_{\mathrm{k}+1}\left(\frac{J_{0}}{2}\right)\right] \cos \left[(2 k+1)\left(2 \pi v m+\phi_{0}\right)\right] .
$$

A similar expression can be obtained for $\Delta \phi_{\mathrm{M}}$.

\subsection{AVERAGING}

To calculate how luminosity changes with time due to random off-set beam-beam interactions, we will need first to average the change $\Delta J_{M}$ and the quadratic quantities $\left(\Delta J_{M}\right)^{2}$ and $\Delta J_{M} \Delta \phi_{M}$. In what follows, we denote the average quantities by the angle brackets, $\left\langle\Delta J_{M}\right\rangle,\left\langle\left(\Delta J_{M}\right)^{2}\right\rangle$ and $\left\langle\Delta J_{M} \Delta \phi_{M}\right\rangle$. Performing the averaging, we will assume that the mean value of the random variable $\zeta_{\mathrm{m}}$ vanishes:

$$
\left\langle\zeta_{m}\right\rangle=0 \text {. }
$$


We also define the auto-correlation function $K(n)$ :

$$
K(n)=\left\langle\zeta_{\mathrm{m}} \zeta_{\mathrm{m}+\mathrm{n}}\right\rangle
$$

This is an even function, $K(n)=K(-n)$, and the setoff variance is simply $\left\langle\zeta_{\mathrm{m}}{ }^{2}\right\rangle=K(0)$.

Consider first $\left\langle\left(\Delta J_{M}\right)^{2}\right\rangle$. The calculations performed in Appendix A give:

$$
\left\langle\left(\Delta J_{M}\right)^{2}\right\rangle=16 \pi^{2} \xi^{2} J_{0} \mathrm{e}^{-J_{0}} \sum_{n=-\infty}^{\infty} K(n) \sum_{k=0}^{\infty}\left(\mathrm{I}_{\mathrm{k}}+\mathrm{I}_{\mathrm{k}+1}\right)^{2} \cos 2 \pi v n(2 k+1) .
$$

The argument of the modified Bessel functions in Eq. (24) is equal to $t 6 / 2$. The derivation of Eq. (24) is based on the assumption that $M \gg 1$ and $v$ is not close to a rational number.

Now we rewrite Eq. (24) in terms of the noise spectral density $S(\omega)$. To define $S(\omega)$, we consider $\zeta$ as a continuous random function of time, $\zeta=\zeta(t)$, with a given auto-correlation function $K(\tau)$ :

$$
K(\tau)=\langle\zeta(t) \zeta(t-\tau)\rangle
$$

According to the spectral theory of random functions, the Fourier transformation of the auto-correlation function gives the spectral density $S(\omega)$ :

$$
\mathrm{K}(\tau)=\int_{0}^{\infty} d \omega S(\omega) \cos \omega \tau
$$

One can conceive that the function $\zeta(t)$ of the continuous time $t$ gives the relative displacement of the bunches about the closed orbit in the course of revolutions. What we need from this function are its values $\zeta_{\mathrm{m}}=\zeta(m T)$ taken at the moments when interactions occur. (Here $T$ denotes the period of revolution, $T=2 \pi / \Omega$, where $\Omega$ is the revolution frequency.) Using the identity

$$
\sum_{n=-\infty}^{\infty} \cos n \alpha=2 \pi \sum_{m=-\infty}^{\infty} \delta(\alpha-2 \pi m)
$$

and changing the order of summations in Eq. (24), one finds

$$
\left\langle\left(\Delta J_{\mathrm{M}}\right)^{2}\right\rangle=16 \pi^{2} \xi^{2} J_{0} \mathrm{e}^{-J_{0}} M \sum_{k=0}^{\infty}\left(\mathrm{I}_{\mathrm{k}}+\mathrm{I}_{\mathrm{k}+1}\right)^{2} R_{\mathrm{k}} .
$$

where the factors $R_{\mathrm{k}}$ are given by

$$
\begin{gathered}
R_{\mathrm{K}} \equiv \sum_{n=-\infty}^{\infty} K(n) \cos 2 \pi v n(2 k+1)= \\
\pi \int_{0}^{\infty} \mathrm{d} \omega \mathrm{S}(\omega)\left\{\sum_{\mathrm{m}=-\infty}^{\infty} \delta[\omega \mathrm{T}-2 \pi m-2 \pi v n(\mathrm{k}+1)]+\sum_{\mathrm{m}=-\infty}^{\infty} \delta[\omega \mathrm{T}-2 \pi m+2 \pi v n(k+1)]\right\}= \\
=\frac{\pi}{T} \sum_{m=0}^{\infty}\left[S\left(\omega_{\mathrm{km}}^{(1)}\right)+S\left(\omega_{\mathrm{km}}^{(2)}\right)\right] .
\end{gathered}
$$


The two sets of frequencies $\omega_{\mathrm{km}}^{(1)}$ and $\omega_{\mathrm{km}}^{(2)}$ are determined by the following equations:

$$
\omega_{\mathrm{km}}^{(1)}=\Omega\{[v(2 k+1)\}+m\}, \omega_{\mathrm{km}}^{(2)}=\Omega\{[1-v(2 k+1)]+m\},
$$

where the brackets denote the fractional par of a number.

The physical meaning of Eq. (28) is that it explicitly expresses the averaged square of $\Delta_{M}$ in terms of the spectral density of the noise. Note that only a discrete set of frequencies enters Eq. (28); these are equal to $\Omega$ multiplied by the distance between $v$ (or its odd harmonic $v(2 k+1)$ ) and the nearest integer. In the next section we will find the contributions of these harmonics to the luminosity dilution.

To finish this section, we calculate $\left\langle\Delta_{M}\right\rangle$. Averaging Eq. (21) with the use of Eq. (22) immediately yields

$$
\left\langle\Delta \Delta_{M}\right\rangle=0 \text {. }
$$

We should emphasize here that this result is valid only in a linear approximation in the parameter $\zeta$. Taking into account second-order terms, generally speaking, gives a non-vanishing contribution to $\left\langle\Delta J_{M}\right\rangle$. However, it is worth noting that in the next section, where we find a perturbation of the distribution function, cnly the first-order averaged value of $\Delta J_{\mathrm{M}}$ enters the result.

Finally, using the same approach as for calculation of $\left\langle\left(\Delta J_{M}\right)^{2}\right\rangle$ the averaging of $\left\langle\Delta J_{M} \Delta \phi_{M}\right\rangle$ is performed in Appendix A. It turns out that $\left\langle\Delta J_{M} \Delta \phi_{M}\right\rangle=0$.

\subsection{DISTRIBUTION FUNCTION OF THE BUNCH}

In this section, we will find the perturbation of the distribution function in the bunch caused by multiple offset beam-beam interactions.

Let us denote by $J_{M}$ and $\phi_{M}$ the values of action and phase after $M$ interactions with an oncoming bunch of a particle which initially had $J_{0}$ and $\phi_{0}$ :

$$
J_{M}=J_{0}+\Delta J_{M}\left(J_{0}, \phi_{0}\right), \phi_{M}=\phi_{0}+\Delta \phi_{M}\left(J_{0}, \phi_{0}\right) .
$$

where $\Delta J_{M}$ is given by Eq. (21). In what follows, we will not need an explicit e.rpression for $\Delta \phi_{\mathrm{M}}$. Since we assume that $\Delta J_{M}$ and $\Delta \phi_{M}$ are small, the Eqs. (32) can be iteratively solved for the initial variables $J_{0}$ and $\phi_{0}$. giving $J_{0}$ and $\phi_{0}$ as functions of the variables $J_{M}$ and $\phi_{M}$. Performing the calculations, we keep terms up to the second order:

$$
J_{0}=J_{M}-\Delta J_{M}\left(J_{M}-\Delta J_{M}, \phi_{M}-\Delta \phi_{M}\right) \approx J_{M}-\Delta J_{M}+\frac{\partial \Delta J_{M}}{\partial J_{M}} \Delta J_{M}+\frac{\partial \Delta J_{M}}{\partial \phi_{M}} \Delta \phi_{M} .
$$

In Eq. (33) it is understood that ihe arguments $J_{0}$ and $\phi_{0}$ in $\Delta J_{\mathrm{M}}$ and $\Delta \phi_{\mathrm{M}}$ are replaced by $J_{\mathrm{M}}$ and $\phi_{\mathrm{M}}$.

Using the property of phase space conservation in Hamiltonian motion, the distribution function after $M$ beam-beam interactions, $F\left(J_{\mathrm{M}}, \phi_{\mathrm{M}}\right)$, can be simply obtained by putting the expression for $J_{0}$ given by Eq. (33) into the initial distribution function $F_{0}\left(J_{0}\right)$. Omitting for convenience the subscripts of variables $J_{\mathrm{M}}$ and $\phi_{\mathrm{M}}$ and using a power series expansion up to the second order, one finds:

$$
F(J, \phi)=F_{0}\left(J_{0}\right)-\left(\Delta J_{M}-\frac{\partial \Delta_{M}}{\partial J_{M}} \Delta_{M}-\frac{\partial \Delta J_{M}}{\partial \phi_{M}} \Delta \phi_{M}\right) \frac{\partial F_{0}}{\partial J}+\frac{1}{2}\left(\Delta J_{M}\right)^{2} \frac{\partial^{2} F_{0}}{\partial J^{2}}
$$


Since we are now keeping second-order terms in our power expansions, $\Delta \mathrm{H}_{M}$ in Eq. (24) is also to be calculated with this accuracy. However, in Sec. 3.0, we have found $\Delta J_{M}$ (Eq. (21)) in the first order only. Slightly changing for a moment our notation, let us denote the right side of Eq. (21) by $\Delta J_{M}^{(1)}$ so that

$$
\Delta J_{\mathrm{M}}=\Delta J_{\mathrm{M}}^{(1)}+\Delta J_{\mathrm{M}}^{(2)}
$$

where $\Delta J_{M}^{(2)}$ is the second-order contribution to $\Delta_{\mathrm{M}}$. As shown in Appendix B, for a Hamiltonian motion, the second-order term can be explicitly expressed through the first-order one:

$$
\Delta J_{\mathrm{M}}^{(2)}=\frac{1}{2} \frac{\partial \Delta J_{\mathrm{M}}^{(1)}}{\partial J} \Delta J_{\mathrm{M}}^{(1)}+\frac{1}{2} \frac{\partial \Delta J_{\mathrm{M}}^{(1)}}{\partial \phi} \Delta \phi_{\mathrm{M}}^{(1)} .
$$

Putting this in Eq. (35), we can find $F(J, \phi)$ given by Eq. (34) through the second order. Now, using the equality

$$
\frac{\partial \Delta J_{M}^{(1)}}{\partial J}+\frac{\partial \Delta \phi_{M}^{(1)}}{\partial \phi}=0
$$

which is a simple manifestation of the phase volume conservation in the first order, and averaging Eq. (34) with account of $\left\langle\Delta J_{M} \Delta \phi_{M}\right\rangle=0$ and $\left\langle\Delta S_{M}\right\rangle=0$, one finds a Fokker-Planck type equation for the averaged perturbation $\Delta F=F-F_{0}$ of the distribution function

$$
\langle\Delta F\rangle=\frac{1}{2} \frac{\partial}{\partial J}\left\langle\left(\Delta J_{M}\right)^{2}\right\rangle \frac{\partial F_{0}}{\partial J} \text {. }
$$

In Eq. (38) we returned to original notation, omit!ing the superscript ${ }^{(1)}$ in $\Delta J_{M}^{(1)}$. Note that $\langle\Delta F\rangle$ depends only on the variable $J$.

\subsection{LUMINOSITY DILUTION}

Substituting Eq. (38) into Eq. (6) and integrating by parts gives a relative change of the luminosity due to offset beam-beam interactions:

$$
\frac{\Delta L}{L_{0}}=-2^{3 / L} \int_{0}^{\infty} d J\left\langle\left(\Delta J_{M}\right)^{2}\right\rangle e^{-3 J / 2}\left[\mathrm{I}_{0}(J / 2)-\mathrm{I}_{1}(J / 2)\right]
$$

Putting Ec. (28) into Eq. (39) and performing numerical integration yields

$$
\frac{\Delta L}{L_{0}}=-\xi^{2} M\left(a_{0} R_{0}+a_{1} R_{1}+a_{2} R_{2}+a_{3} R_{3}+\ldots\right)
$$

where the coefficients $a_{\mathrm{k}}$ are

$$
a_{0}=12.45, a_{1}=0.73, a_{2}=0.038, a_{3}=0.002 .
$$

As we see, the largest cocfficient in the series (40) is $a_{0}$; for large $k, a_{\mathrm{k}}$ rapidly fall down. The factor $R_{0}$ corresponding to $a_{0}$ contains different harmonics of the noise spectrum. In a typical case, the noise 
spectrum is a decreasing function of $\omega$, and the main contribution to $R_{0}$ comes from the first term in Eq. (29):

$$
R_{0}=\frac{\pi}{T} S\left(\Omega v v^{\prime}\right)
$$

where $\Delta v$ is the distance from $v$ to the nearest integer. Depending on the working tunes, the next term in Eq. (40) proportional to $a_{1} R_{1}$ may also be impontant because $R_{1}$ contains different harmonics of the noise.

\subsection{DISCUSSION}

To understand the rolc of the effects under study in the SSC, let us estimate a tolerable setoff of the beams at the interaction point.

Note that according to Eqs. (25) and (26) the spectral density $S$ has a dimension of the length squared over the frequency. Hence, the product $\Omega \Delta v S(\Omega \Delta v)$, which has a dimension of length squared, can be associated with the beam displacement squared, $\delta x^{2}$, at the frequency $\Omega \Delta v$. In terms of $\delta x$, Eq. (40) takes the form

$$
\frac{\Delta L}{L_{0}}=-6.2 \xi^{2} M \frac{\delta x^{2}}{\Delta v} .
$$

Now, putting in Eq. (43) $\Delta v=0.26$ and $\xi=0.001$ (for one interaction point) and requiring $\Delta L / L_{0}$ to be less than 0.5 after 10 hours of operation $\left(M=10^{8}\right)$, we obtain:

$$
\delta x<1.1 \times 10^{-2} \sigma_{x} .
$$

In Eq. (44) we retumed to dimensional variables, putting $\sigma_{x}$ on the right side. For $\sigma_{x}=5 \mu \mathrm{m}$ at the low beta interaction point, this gives a serious constraint $\delta x<5.5 \times 10^{-2} \mu \mathrm{m}$ on a possible beam offset. 


\section{ACKNOWLEDGMENTS}

I would inke to thank Prof. A. N. Skrinsky, who stimulated this work, for many illuminating disrussions. 


\section{REFERENCES}

1. Site Specific Conceptual Design Report, SSCL-SR-1056.

2. F.M. Izrailev, Proc. of the Third Advanced ICFA Beam Dynamics Workshop, Novosibirsk, 1989, p. 92. 


\section{APPENDIX A}

Let us first calculate $\left\langle\left(\Delta J_{M}\right)^{2}\right\rangle$. Averaging $\left(\Delta J_{M}\right)^{2}$ and using Eq. (23), one finds

$$
\begin{aligned}
& \left(\Delta J_{M}\right)^{2}=32 \pi^{2} \xi^{2} J_{0} e^{-J_{0}} M \sum_{l, m=0}^{M-1} K(m-l) \times \\
& \times \sum_{k=0}^{\infty}\left[h_{k}+I_{k-1}\right] \cos \left[(2 k+1)\left(2 \pi v m+\phi_{0}\right)\right] \times \\
& \times \sum_{r=0}^{\infty}\left[L_{l}+L_{1-1}\right] \cos \left[(2 r+1)\left(2 \pi v l+\phi_{0}\right)\right],
\end{aligned}
$$

where the argument of the modified Bessel functions is equal to $J_{0} / 2$. Now define a new summation index, $n=m-l$, and change the order of summation using the identity

$$
\sum_{m=0}^{M-1} \sum_{n=m-M+1}^{m}=\sum_{n=1}^{M-1} \sum_{m=n}^{M-1}+\sum_{m=0}^{M-1}\{n=0\}+\sum_{n=-M+1}^{-1} \sum_{m=0}^{n+M-1}
$$

After this, one can sum up over $m$ because this index enters cosine arguments only. Then, using elementary trigonometric identities for the product of two cosines, we obtain a formula that contains the following expressions:

$$
\sum_{m} \cos \left\{2 \pi v m[(2 r+1) \pm(2 k+1)]-2 \pi v n(2 r+1)+[(2 r+1) \pm(2 k+1)] \Phi_{0}\right\},
$$

where the number of additives in ( $\mathrm{A} 3$ ) is equal to $M-n$. Assume that $v$ is not a rational number. In this case, for $r \neq k$ the sum (A3) is of the order of unity even in the limit $M * 1$, and only for $r=k$ is this sum equal to

$$
(M-n) \cos 2 \pi v n(2 k+1)=M \cos 2 \pi v n(2 k+1),
$$

that is, it grows linearly with $M$. In (A4) we took into account that $M * n$, because $n$ is, in fact, restricted from above by $K(n) \rightarrow 0$ for large $n$.

Keeping only the largest terms (proportional to $M$ ) in Eq. (A1), one finds

$$
\left\langle\left(\Delta U_{M}\right)^{2}\right\rangle=16 \pi^{2} \xi^{2} J_{0} \mathrm{e}^{-J_{0}} M \sum_{n=-M-1}^{M-1} K(n) \sum_{k=0}^{\infty}\left(\mathrm{I}_{\mathrm{k}}+\mathrm{I}_{\mathrm{k}+1}\right)^{2} \cos 2 \pi v n(2 k+1) .
$$

Since the sum over $n$ in (A5) converges when $|n| \rightarrow \infty$. one can extend summation from $-\infty$ to $\infty$. Using the evenness of the function $K(n)$, we arrive at Eq. $(24)$.

Using the same approach as above. one can calculate the averaged value of $\left\langle\Delta J_{M} \Delta \phi_{M}\right\rangle$. The result is

$$
\left\langle\Delta J_{\mathrm{M}} \Delta \phi_{\mathrm{M}}\right\rangle=8 \pi^{2} \xi^{2} J_{0} \mathrm{e}^{-J_{0}}, \sum_{n=-11-1}^{M-1} K(n) \sum_{k=0}^{\infty}\left(\mathrm{I}_{\mathrm{k}}+\mathrm{I}_{\mathrm{k}+1}\right)^{2} \sin 2 \pi v n(2 k+1) .
$$

However this is equal to zero because the product $K(n) \sin 2 \pi v n(2 k+1)$ is an odd function of $n$. 
APPENDIX B

Let us consider a time-independent Hamiltonian $H(p, q)$ with the corresponding Hamiltonian equation:

$$
\frac{d p}{d t}=-\frac{\partial H}{\partial q}, \quad \frac{d q}{d t}=\frac{\partial H}{\partial p} .
$$

Let $p_{0}$ and $q_{0}$ denote initial values of the phase variables. Let us consider a motion in which $p$ and $q$ change by small values, $\Delta p$ and $\Delta q$. In the first approximation, they can be found by integrating Eq. (B1) over time and putting in the Hamiltonian $p_{0}$ and $q_{0}$ instead of $p$ and $q$ :

$$
\begin{aligned}
& \Delta p^{(1)}=-\int_{\mathrm{t}_{0}}^{\mathrm{t}} \frac{\partial H\left(p_{0} . q_{0}\right)}{\partial q_{0}} d t^{\prime}=-\left(t-t_{0}\right) H_{q}\left(p_{0}, q_{0}\right) . \\
& \Delta q^{(1)}=\int_{\mathrm{t}_{0}}^{t} \frac{\partial H\left(p_{0}, q_{0}\right)}{\partial p_{0}} d t^{\prime}=\left(t-t_{0}\right) H_{p}\left(p_{0}, q_{0}\right) .
\end{aligned}
$$

where the indices $p$ and $q$ denote parial derivatives with respect to the corresponding variables.

In the next approximation, while integrating (B1) one should take into account that $p$ and $q$ differ from $p_{0}$ and $q_{0}$ in the first order:

$$
\frac{d p}{d t}=-H_{q}\left(p_{0} \cdot q_{0}\right)-H_{q q}\left(p_{0} \cdot q_{0}\right) \Delta q^{(1)}-H_{q p}\left(p_{0} \cdot q_{0}\right) \Delta p^{(1)}
$$

Integration of Eq. (B4), taking into account Eqs. (B2) and (B3), now yields

$$
\Delta p=\Delta p^{(1)}+\frac{1}{2} \frac{\partial \Delta p^{(1)}}{\partial q} \Delta q^{(1)}+\frac{1}{2} \frac{\partial \Delta p^{(1)}}{\partial p} \Delta p^{(1)} .
$$

One can easily check that the result (B5) is also valid for a Hamiltonian $H$ depending versus time through a factor $f(t)$,

$$
H(p, q, t)=f(t) H_{0}(p, q) .
$$

This is just the case relevant to the problem of beam-beam interaction, for which the Hamiltonian is given by Eq. (12) with the time variable equal to $\theta$. Rewriting Eq. (B5) in terms of action and phase, we arrive at Eqs. (36). 

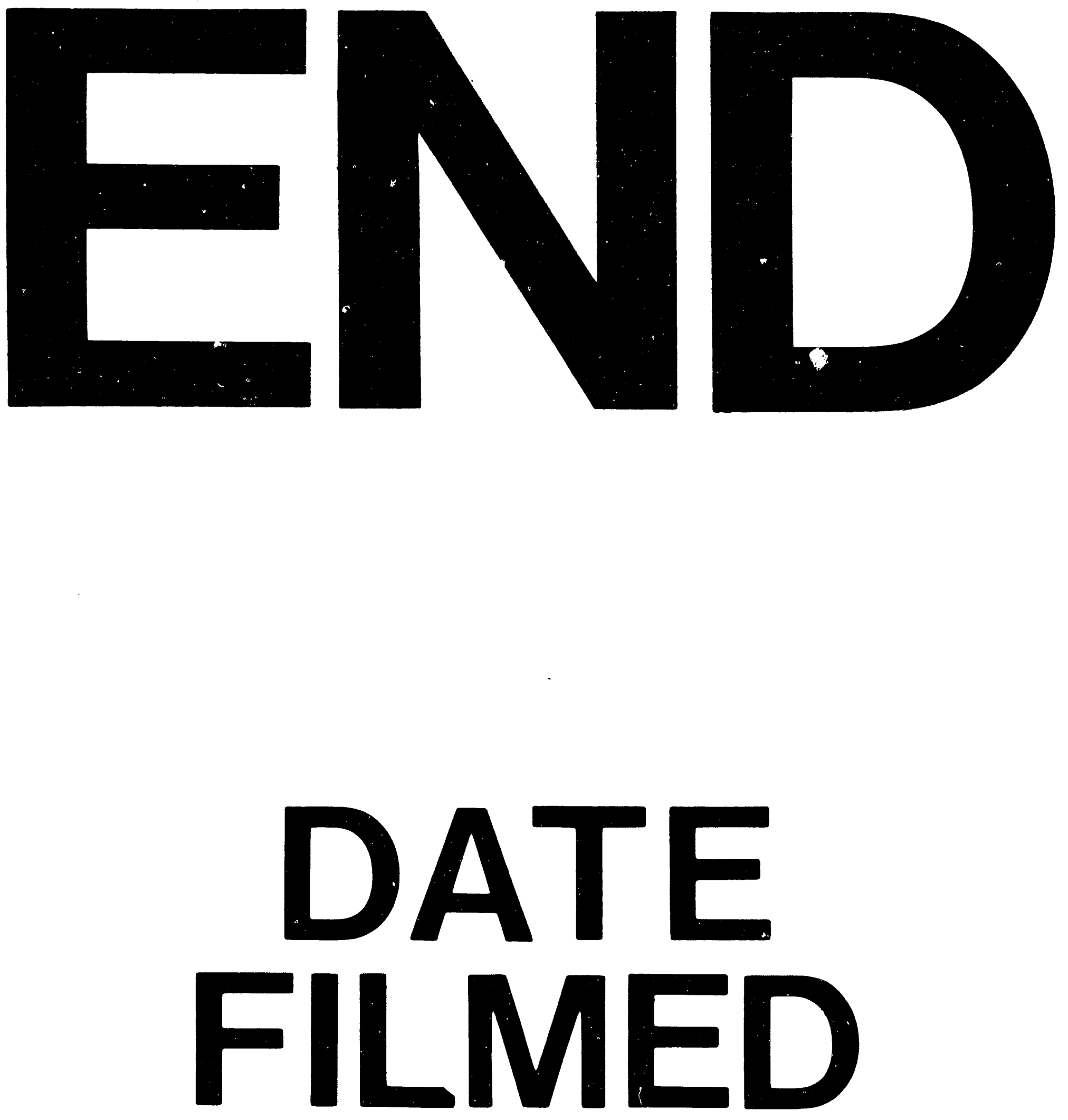

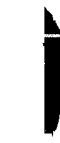

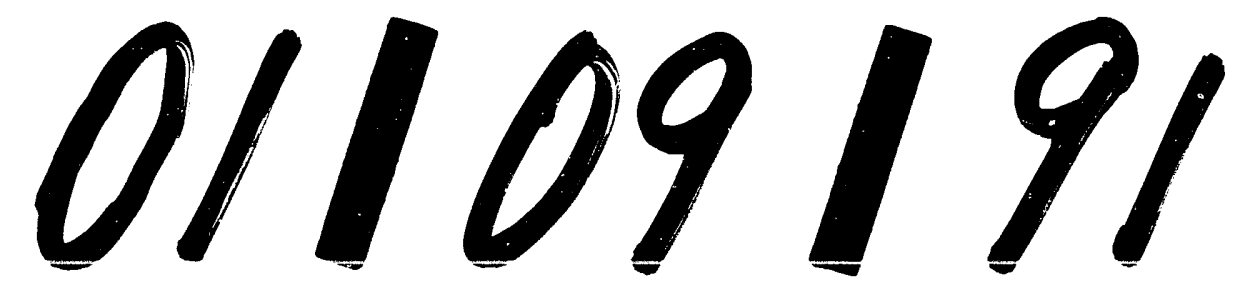

- 
